\title{
Formación y Gestión de Desempeño del Docente- Investigador en la Educación Superior: Modelo Teórico Basado en Competencias
}

\section{Guillermo Terán}

Resumen-El presente trabajo tiene como objetivo fundamental generar un modelo teórico de la formación y gestión del desempeño del docente-investigador en la Educación Superior. Basado en competencias, el cual permitirá orientar la gestión de la calidad en el aprendizaje, la investigación y la docencia universitaria.

Con la finalidad de lograr el propósito se procedió a diagnosticar, entre otros aspectos, las competencias que debe tener el docente universitario en el desarrollo académico.

Fundamentados teóricamente en concepciones sobre educación superior basados en aprendizajes durante toda la vida, con capacidad de movilizar un conjunto de recursos -saberes, saber-ser y saber hacer- en un contexto ligando conocimientos, capacidades y comportamientos. La metodología empleada se apoyó en una investigación cualitativa interpretativa. Los hallazgos del trabajo permitieron generar un modelo teórico orientado a fortalecer el desarrollo de competencias investigativas para potenciar el perfil y desempeño profesional del docenteinvestigador universitario, encaminado al logro de cambios profundos y significativos que coadyuven el mejoramiento en la calidad de la Educación Superior.

Abstract - The fundamental aim of this work is to generate a theoretical model of the formation and management of the performance of the professor - investigator in the higher education.

This model will be based on competitions, which will allow to orientate the quality management in the learning process, investigation and University teaching. In order to reach that purpose, it was decided to diagnose, between other aspects, the competences that must have a professor in the academic development, which must be theoretical founded in conceptions about top education based on learning acquired through life. Also, they are able to mobilize resources - knowledge to know to be and do, in a context joining knowledge, capacities and behaviors.

The used methodology rested on a qualitative interpretative investigation. The results allow to generate a theoretical model orientated to strengthening the development of investigative competitions. In order to promote the profile and professional performance of the professor - investigator, directed to the achievement of deep and significant changes that contribute to the improvement of the quality of the Higher Education. 
Palabras clave- Formación, gestión de desempeño, docenteinvestigador, competencias, educación superior.

\section{Introducción}

Hoy en día la sociedad y todas sus instituciones, particularmente la educación superior, debe enfrentar retos difíciles como el formar profesionales cualificados capaces de generar conocimiento y conducir cambios en la sociedad. Frente a estas nuevas demandas y desafíos, las instituciones de educación superior funcionan y se desarrollan en ambientes económicos, sociales, políticos y culturales extremadamente complejos; están adquiriendo nuevas y variadas funciones -signados porel cambio constante, la paradojay la incertidumbrecambios en la vida institucional, en las estructuras, en los estilos de organización y en el papel social y económico, característica, de las universidades contemporáneas.

La universidad como una organización social, acepta la definición de sistema social abierto, que incorpora recursos de su entorno, procesa esos recursos de una cierta manera y, los devuelve convertidos en determinado producto; reincorpora luego nuevos recursos para reenergizarse y mantenerse en el tiempo. Picón (1994).

La universidad es una institución que utiliza como materia prima, una"sustancia"sumamente variable en sí misma y muy sensible a los cambios y presiones provenientes de los más diversos sectores de la sociedad, como es el conocimiento.

Para cumplir con la misión y objetivos, la universidad aplica al conocimiento, una gama de tecnologías que históricamente se han venido agrupando en tres grandes conjuntos de actividades: docencia, investigación y extensión.

La universidad como institución social, propone una visión estratégica para construir un nuevo desarrollo, debe asumir que los tiempos que vive ella misma, el Estado, el país y el mundo son tiempos de cambio.

Todo ello trae consigo un amplio debate sobre el futuro de la educación superior y la generación de propuestas alternativas que marquen una visión distinta.

\section{En este sentido:}

La misión de la educación para la era planetaria es fortalecer las condiciones de posibilidad de la emergencia de una sociedadmundo compuesta por los ciudadanos protagonistas, conscientey críticamente comprometidos en la construcción de una civilización planetaria (Morin, 2003: 122).

Ante esta compleja realidad, los temas abordados dentro de los debates destaca el de los sistemas educativos y la necesidad de revisarlos y transformarlos para enfrentar demandas de una nueva naturaleza, asociadas al mundo globalizado en el que se encuentran insertas las sociedades. (Barrón, T. 2009). En la sociedad del conocimiento la importancia del capital humano radica en que es una fuente de creación de ventajas que proceden, producto de la formación integral, la capacidad creativa, la producción de conocimiento mediante la investigación, la habilidad para identificar y resolver problemas, liderar y gestionar eficientemente organizaciones productoras de indicadores sociales. La universidad se constituye en el espacio estrategico y privilegiado de generación del conocimiento de la sociedad.

Delors (1996), en el informe presentado a la Organización de las Naciones Unidas para la Educación, la Ciencia y la Cultura (UNESCO) con la Comisión Internacional sobre Educación para el siglo XXI, platean la adopción de un rol alternativo del docente de educación superior, acorde con las demandas de una nueva sociedad mundializada-globalizada, capaz de apoyar el desarrollo integral del estudiante; esto implica el compromiso de la universidad el de posesionar una nueva vision, misión y valores $y$, un nuevo paradigma para la formación de los futuros profesionales, basados en el aprendizaje para la vida, la orientación prioritaria hacia el aprendizaje autogestionario e independiente -aprender a aprender, aprender a emprender y aprender a ser- y el diseño de estrategias y metodologías educativas alternativas donde el estudiante se constituya en el actor central del proceso académico.

En concordancia con lo expresado, el presente artículo se inscribe en dichos procesos, como respuesta a los requerimientos de la sociedad y la búsqueda de soluciones a problemas reales. El abordaje de este estudio se realiza mediante un primer acercamiento con la realidad, donde se contextualiza el rol del docente-investigador en la educación superior, apoyado en una metodología cualitativa interpretativa que permite identificar indicadores y perspectivas que tienen los actores (docentes universitarios) en su formación y gestión de su desempeño profesional basado en competencias.

Dichos hallazgos permitieron generar un cuerpo estable de ideas relacionados con las competencias formativas y de gestión del desempeño del docente-investigador que demanda la sociedad actual, desde una estructura epistemológica-filosófica, humanista, socio-crítica.

\section{Caracterización Teórico-Conceptual de Competencias del Docente Desde una Visión Dinámica}

El enfoque de la educación basada en competencias se ha convertido en pocos años en la orientación central alrededor de la cual gira la gestión de la calidad en la educación 
superior con la finalidad de dar respuesta a los requerimientos que exige la sociedad actual. Esto implica concebir a la educación superior de forma diferente, basados en aprendizajes durante toda la vida, con capacidad de movilizar un conjunto de recursos -saberes, saber-ser y saber hacer- en un contexto definido articulando conocimientos, capacidades y comportamientos.

En relación con esta perspectiva, en el marco de acción para el cambio y el desarrollo de la educación superior, se producen movimientos transformadores en países de la Unión Europea y América Latina, mediante la génesis de, proyectos tales como el Proyecto Tuning de la Unión Europea, el proyecto Alfa Tuning de Latinoamérica y el Proyecto "6 x 4", entre otros. De ahí la importancia del estudio en profundidad de esta área temática.

En consecuencia, se considera de suma importancia establecer un proceso inter-relacionador entre aprendizajes y competencias que se ajusten a lineamientos orientadores del rol del docente-investigador en su desempeño profesional y formación.

Así, Draegert, M., (2002), conceptualiza a las competencias como el conjunto de saberes intergrados en la actividad intelectual y creadora del docente que reflexiona sobre la práctica cotidiana y actúa para mejorar la calidad de su propia intervención.

En este orden de ideas, y en el proceso integrador aprendizaje y competencias, se tiene las a) Competencias del conocer: que comprende las aptitudes, habilidades y destrezas que incluyen los procesos conceptuales, la metacognición, los procesos de desarrollo del pensamiento, la lógica, el análisis, la reflexión, la interpretación, la inferencia y la generalización de reglas, principios y métodos. Por su intermedio, se reconocen y comprenden las ideas, nociones abstractas, imágenesy símbolos; b) Competencias del aprender a hacer: Más allá del aprendizaje de una profesión, conviene, en un sentido más amplio, adquirir competencias que permitan enfrentar nuevas situaciones y faciliten el trabajo en equipo y brinde la posibilidad de movilizarse dentro del área ocupacional; c) Competencias del aprender a convivir: Implican aprender a desarrollar el conocimiento personal aceptando el enriquecimiento proveniente de los saberes y experiencias de los demás y brindando los propios, de modo que se cree una nueva mentalidad, la cual -basada en la aceptación de nuestra mutua interdependencia y en los riesgos y desafíos del futuro- impulse la ejecución de proyectos comunes cuyo objetivo involucre el mejoramiento de la calidad de vida. Estas competencias demandan participación activa y consciente en acciones comunitarias, actividades culturales, científicas y empatía con otras culturas; d) Competencias del aprender a ser: El siglo XXI exige a todos una mayor capacidad de autonomía y de juicio, que va a la par del fortalecimiento de la responsabilidad personal en el destino de la humanidad. Se aspira a la formación de un individuo analítico, crítico, creativo, espontáneo, libre, sensible, curioso e imaginativo, autónomo, con espíritu de investigación, exploración y cuestionamiento, que aprenda por sí mismo, comprenda y transmita significados.

Estas competencias humanísticas se operacionalizan con el desarrollo de las competencias investigativas durante el proceso de formación y ejercicio profesional del docente.

\section{Visión Multidimensional del Modelo Teórico de Formación y Desempeño del Docente-Investigador en Educación Superior Basado en Competencias}

\section{Competencias profesionales del docente-investigador}

La formación y al rol del docente-investigador se encuentran estrechamente vinculados con los cambios culturales, científicos, educativos, sociales, políticos y económicos que están impactando a todas las sociedades. Como respuesta a dichos cambios, el docente-investigador debe ampliar su rol en función de las necesidades de aprendizaje y del entorno en los que se desarrollará apoyados en el uso de las tecnologías de la información y comunicación TICs., de los estructuras culturales y de las comunidades universitarias, entre otros aspectos.

Para cumplir con éste propósito se demanda al docente-investigador desarrollar competencias relacionadas con el dominio de idiomas, el manejo de tecnologías de la información y comunicación TICs. (saberes), el desarrollo de competencias inter-relacionales, comunicacionales o sociorelacionales (saber ser) y el manejo de las relaciones humanas (manejo de talentos humanos y trabajo en equipo), así como una cuerpo de requerimientos de estrategias y metodologías (saber hacer), direccionados a la aplicación de los conocimientos a situaciones concretas y reales.

La competencia profesional del docente-investigador, que de acuerdo a la perspectiva del autor, es una competencia intelectual, trasciende el sentido eminentemente técnico del recurso académico. Las competencias del docenteinvestigador se caracterizan por ser multidimensionales: integran holísticamente conocimientos, habilidades, principios y comportamientos direccionadas a las prácticas investigativas; así como una reflexión y análisis critico sobre los contextos que las condicionan y que van más allá del claustro universitario. La competencia profesional (Contreras, 1999: 58). "... se refiere no sólo al capital de conocimientos disponibles, sino a los recursos intelectuales de que dispone con objeto de hacer posible la ampliación y desarrollo de ese conocimiento profesional, su flexibilidad y profundidad". 
La docencia universitaria aparece así ligada a un conjunto de competencias investigativas, pedagógicas, didácticas, entre otras, en cuya estructura y funcionalidad juegan un importante papel el conocimiento teórico-práctico y la actividad crítico-reflexiva sobre la práctica. Perrenoud (2007) señala que el concepto de competencia representa la capacidad de movilizar varios recursos cognitivos para saber hacer frente a un determinado tipo de situaciones.

Las competencias por si solas no son conocimientos, habilidades o actitudes -aunque movilizan varios recursos cognitivos- dicha movilización es única, es decir, contextualizada en situaciones y entornos específicos. El desarrollo de la competencia pasa "por operaciones mentales complejas, sostenidas por esquemas de pensamiento", (Perrenoud, 2007: 11) y finalmente las competencias profesionales se crean en el proceso de formación como en la gestión del ejercicio profesional. En éste sentido se concibe a la competencia como un constructo fundamental que se refiere a un conjunto de conocimientos y habilidades que los seres humanos requieren para desarrollar algún tipo de actividad. Cada actividad exige un número variado de competencias que pueden ser desglosadas en unidades más específicas de competencia en las que se especifican las tareas concretas que están incluidas en la competencia global. Se puede manifestar que la competencia está formada por diversas unidades de competencia. (Barrón, T. 2009: 76-87)

\section{Competencias investigativas}

En el ámbito de la investigación la presencia del docente-investigador alude a la del investigador que se responsabiliza académicamente del estudiante de manera inmediata, directa y permanente; establecen una relación de trabajo y crean un proyecto de formación específico para el estudiante (Sánchez y Santa María, 2000). Es en este espacio mediado por múltiples situaciones en donde se pone en juego la formación del futuro investigador.

En este orden de ideas, la docencia en la educación superior se apoya en un conjunto de competencias investigativas, andragógicas y didácticas, en cuya estructura y funcionalidad juegan un importante papel las competencias de los saberes teórico-epistemológicos, saberes metodológico-prácticos y los saberes aplicativos critico-reflexivos; pilares directrices del modelo teórico que el docente-investigador debe cumplir para generar conocimiento en las diferentes áreas del saber mediante la investigación.

a) Competencias de saberes teórico-epistemológicos: Esta competencia es inherente al conocimiento teórico que el docente-investigador debe conocer sobre: epistemología, en el sentido de la consideración de nuevas formas de producir conocimiento; ontología, en cuanto a la conceptualización del tipo de hombre que demandan los nuevos tiempos; axiología, en tanto que supone el surgimiento de una nueva escala de valores; paradigmas, conceptualizado desde una perspectiva de pluralidad de significados y diferentes usos, como: "Una red de creencias teóricas y metodológicas, valores, ideas, técnicas y procedimientos compartidos por una comunidad científica para solucionar problemas"; supuestos teóricos, definidos como complejos creados mediante el desarrollo de un conjunto de proposiciones o generalizaciones interrelacionadas de algún modo sistemático; marcos conceptuales; conocimientos y diseños reconocidos socialmente por la comunidad científica. Dicha teorías, sistemas conceptuales y orientaciones filosóficasepistemológicas van indisolublemente unidos en todas las fases de una investigación con independencia de si el hecho de que se utilicen sea consciente y explícito o inconsciente e implícito.

b) Competencias delos saberes metodológico-prácticos:conforman el conjunto de estrategias, habilidades y destrezas propias de la actividad del docente-investigador. A través de este tipo de saberes se enseña y se aprende a problematizar, a fundamentar la teoría, a concebir la teoría como una herramienta de análisis, a organizar, analizar e interpretar la información. Cada uno de estos quehaceres de la investigación está integrado por numerosas y diferentes operaciones que requieren conocimientos teóricos, habilidades y estrategias.

La formulación inicial explícita de una cuestión o problema que investigar determina el tema de interés y fija los parámetros del resto de las áreas de decisión.

El docente-investigador debe explicitar el marco teórico de su trabajo. Pero hacerlo supone algo más que ofrecer una revision de la bibliografía; toda vez que la investigación es un proceso interactivo de conducta y pensamiento (Denzin, 1994), guiado sistemáticamente por las reglas del método científico y del razonamiento lógico, así como por los sistemas de marcos teóricos y conceptuales compartidos por las comunidades de expertos.

El método científico, es el procedimiento o conjunto de procedimientos que se utilizan para obtener conocimientos científicos, el modelo de trabajo o secuencia lógica que orienta la investigación científica (Sabino, C. 2002). El estudio del método o de los métodos, si se quiere dar al concepto un alcance más general se denomina metodología, y abarca la justificación y la discusión de su lógica interior, el análisis de los diversos procedimientos concretos que se emplean en las investigaciones y la discusión acerca de sus características, cualidades y debilidades.

Sin embargo en el lenguaje cotidiano del mundo académico, la palabra metodología se utiliza también muy extensamente en sentidos diferentes, opuestos a veces al anterior: se habla así de "metodología de la investigación" 
para hacer referencia a los pasos y procedimientos que se han seguido en una indagación determinada, para designar modelos concretos de trabajo que se aplican en una disciplina o especialidad y también para hacer referencia al conjunto de procedimientos y recomendaciones que se transmiten al estudiante como parte de la docencia en estudios superiores.

También suelen designarse como métodos a los estilos de trabajo peculiares de cada disciplina como: el método antropológico y a las formas particulares de investigación que se utilizan para resolver problemas específicos de indagación, como el método cualitativo, el método experimental o el método estadístico, entre otros.

Además, el docente-investigador requiere manejar las tecnologías de la información y de la comunicación TICs. (Internet, correo electrónico, multimedia, videoconferencia), lo que implica un cambio de roles: se requiere de un docenteinvestigador que mantenga contacto interactivo permanente con los estudiantes a través de la red, de manera que su práctica se centrará más en ayudar y orientar al estudiante para la selección, organización e interpretación de la información y contenidos de las mismas, la adquisición de habilidades y el intercambio de información y de experiencias con sus pares nacionales e internacionales.

c) Competencias delos saberes aplicativos critico-reflexivos:estetipo de saberes se refiere a los significados y propósitos propiamente dichos del conocimiento que se generan, mediante procesos de profunda reflexión y criticidad. El docente-investigador debe cuestionarse acerca de los fines últimos de la investigación, los por qué de carácter ético-profesional del saber científico, así como los para quiénes de la producción científica (Sabino, C. 2002). Esto implica retomar la labor teórica para poder obtener de ellos la respuesta al problema planteado: será preciso analizar críticamente la información, proceder a sistematizarla, sintetizarla, y arribar a conclusiones globales, generadoras de grandes políticas.

Estamos, así, en el momento final de la investigación. Con esto se cierra, aunque sólo parcialmente, el proceso de investigación, por cuanto ninguna investigación resuelve completamente los problemas formulados. (ob. cit.) Generalmente los hallazgos de la investigación generan respuestas parciales, en el mejor de los casos, la respuesta eficaz de un problema implica la aparición de varios otros nuevos problemas a investigar. El ciclo se reiniciará, de un modo circular e inacabable, como lo es en verdad la tarea del hombre por resolver los interrogantes del mundo que lo rodea y de su propia práctica.

Lo que aquí hace la diferencia no es propiamente la naturaleza de la investigación, sino el uso que se le da a la misma, para lo cual se requiere del compromiso de ambos actores, el estudiante y el docente-investigador, insertos en un espacio académico en donde se establecen lazos particulares a través de las relaciones diarias, los vínculos, la delimitación de identidades, expectativas, ritos, mitos y creencias, etc.

Finalmente, se puede expresar que los nuevos perfiles generados por las exigencias de un mundo globalizado requieren a su vez de una reorientación y de actualizar contenidos, metodologías, estrategias y formas de trabajo en el salón de clase universitario. Como parte imprescindible de esta formación la UNESCO señala que la educación superior del futuro deberá integrar valores como la solidaridad, la mística del trabajo humano, la responsabilidad, los derechos humanos, el respeto a la paz y al entorno y la consolidación de la identidad cultural y social.

Indudablemente los recursos humanos que se han movilizado para enfrentar el reto de la formación y gestión del docente-investigador son muy variados; lo que se puede rescatar como un denominador común es el deseo de conocer a qué responde este nuevo rol profesional, cuáles son las funciones que se tienen que desempeñar. Cambiar el rol y el profesionalismo del docente-investigador sólo puede hacerse promoviendo una reforma de pensamiento con una visión del mundo más acorde con las necesidades del planeta y las aspiraciones humanas.

\section{Bibliografía}

[1] Barrón, C. y Gutiérrez, N. (2002), La formation des tuteurs pour la recherche en sciences sociales dans le mexique d'aujourd'hui: analyse des strategies, en Congres De L'afirse, Pau, Francia, pp. $1-15$.

[2] Barrón, T. (2009), "La teoría de las generaciones como una categoría analítica de estudio en la formación del profesional universitario", en A. Díaz Barriga (coord.), Currículo, evaluación y planeación educativa, México, COMIE, pp. 119-132

[3] Contreras, José (1999), La autonomía del profesorado, Madrid, Morata.

[4] Castells, M. (1996).VReunión Nacional de Currículo"Escenarios para la Universidad del Siglo XXI". La Implantación de las Nuevas Tecnologías en las Universidades. UCV.

[5] Denzin, N. (1994). Handbook of qualitative research, Sage, Thousand Oaks C. A.

[6] Didriksson, A. (1995). Una Agenda del Presente para la Construcción del Futuro de la Educación Superior en América Latina y el Caribe. En: La UNESCO Frente al Cambio de la 
Educación Superior en América Latina y el Caribe. Memoria del Seminario UNAM / UNESCO, México, junio de 1995. Cresalc / UNESCO.

[7] Draegert, M., (2002). Docencia universitaria y competencias didácticas. Perfiles Educativos, Vol. XXXI, Núm. 125, 2009, pp. 7687.

[8] Gimeno, José (1995), "Paradigmas crítico-reflexivos en la formación de profesores. Derivaciones para los contenidos y para las prácPerfiles Educativos.Vol. XXXI, núm. 125, 2009. IISUEUNAM

[9] Gerstner, L., (1996). Reinventando la Educación: Nuevas formas de gestión educativa. Barcelona: Paidos.

[10] González, C., (2000). Competencias y Proyecciones de la Formación Docente en Preescolar. Ponencia presentada en el Congreso internacional de Pedagogía Alternativa. Barquisimeto Venezuela

[11] Latorre, A. (2003). La Investigación Acción y el Cambio Organizacional. Capítulo 11 del texto Estrategias de Formación para el Cambio Organizacional. Barcelona, España: Editorial EDO. $526 \mathrm{p}$.

[12] Morín, E. (1999). La cabeza bien puesta. Buenos Aires, Argentina: Ediciones Nueva Visión.

[13] (2000). Los Siete Saberes Necesarios para la Educación del Futuro. Bogota. Ministerio de Educación Nacional.

[14] Morin, E., Roger, E. y Motta, R. (2003), Educar en la era planetaria, Barcelona, Gedisa.

[15] Paez, H. (1999). Perfil de Competencias del Docente de Educación Superior Hacia y en el Nuevo Milenio. Trabajo presentado en el encuentro por la unidad de los Educadores Latinoamericanos. Pedagogía 1999. Universidad del Zulia Programa Doctoral en Ciencias Humanas (2004). Línea de investigación. Tecnología y Currículo. Maracaibo, Zulia, Venezuela.

[16] Peiró,J., (sf). Las competencias en la sociedad de la información: nuevos modelos formativos. Universidad de Valencia disponible en: http://cvc.cervantes.es/obref/ formacion_virtual/formacion_continua/

[17] Perrenoud, P. (2007), Diez nuevas competencias para enseñar, México, Editorial Graó.

[18] Picón, G. (1994). El proceso de convertirse en universidad: Aprendizaje organizacional en la universidad Venezolana.
Caracas, Venezuela: Fondo Editorial de la Universidad Pedagógica Experimental Libertador.

[19] Piña Osorio, J. M. y O. Mireles (2000), "El proceso de socialidad y de vida académica", en R. Sánchez Puentes y M. Arredondo (coord.), Posgrado en Ciencias Sociales y Humanidades. Vida académica y eficiencia terminal, México, CESU-UNAM, pp.79108.

[20] Orozco, D. (2000). Perfiles de los Educadores y las Educadoras y la Formación Permanente de Cara al Nuevo Milenio. Costa Rica.

[21] Rivas, C. (1996). Un nuevo paradigma en educación y formación de recursos humanos.Cuadernos Lagovén. Caracas: Editorial Arte.

[22] Sabino, C. (2002). Cómo Hacer una Tesis, Op. Cit., caps. 9 y 10 .

[23] Salcedo, H. (1999). Perfeccionamiento integral y evaluación del Profesorado universitario. Trabajo presentado en el Primer Encuentro Iberoamericano de Perfeccionamiento Integral del Profesor Universitario. Caracas. Universidad Central de Venezuela.

[24] Sánchez, R. y Santa María, M. (2000), "El proceso y las prácticas de tutoría", en Ricardo Sánchez Puentes y Martiniano Arredondo (coord.), Posgrado en Ciencias Sociales y Humanidades. Vida académica y eficiencia terminal, México, CESU-UNAM, pp. 109136.

[25] Tunnermann, C. (1999). Realidad y perspectiva de la universidad en el contexto Latinoamericano. Revista Prospectiva. Publicación Semestral del Núcleo de Directores de Planificación de las Universidades. Venezuela.

[26] (2002). Tendencias en la transformación de la educación superior. V Reunión Nacional de Currículo: Escenarios para la Universidad del Siglo XXI. Caracas: CNU. UCV. NVA. CCN. Caracas. Venezuela.

[27] UNESCO (1996). Informe de la Comisión Internacional sobre la Educación para el Siglo XXI. La educación encierra un tesoro. España: Editorial Santillana.

[28] (1998). Informe a la UNESCO de la Comisión Internacional sobre la Educación de Adultos. Madrid, España: Editorial Santillana.

[29] (1999). Informe a la UNESCO de la Comisión Internacional sobre la Educación para el Siglo XXI. Madrid, España: Editorial Santillana. 
[30] (1998). Plan de acción para la transformación de la educación superior en América Latina y el Caribe. Caracas, Venezuela: CRESALC.

[31] Zabalza, Miguel A. (2003), Competencias docentes del profesorado universitario. Calidad y desarrollo profesional, Madrid, Narcea.

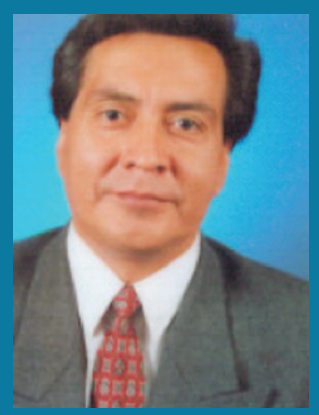

Guillermo Terán. Se graduó en la Universidad Central del Ecuador en Física y Matemática 1980; UniversidadTécnica de Ambato doctor en Planificación e Investigación 1998; estudió en la Universidad Pedagógica Experimental Libertador UPEL de Caracas-Venezuela se graduó de magister en educación superior: mención gerencia. 1996. Obtuvo el Título de doctor Ph.D en educación e investigación en UPEL Caracas-Venezuela. 2.004

Ejerció profesionalmente en las áreas de investigación, matemática, física, informática e investigación en la Universidad Pedagógica Experimental Libertador y en el Instituto Universitario Tecnológico de CaracasVenezuela. Fue supervisor académico e investigador en la Escuela Superior Militar Eloy Alfaro. Actualmente es docente de la Universidad Central del Ecuador en Física, Sistemas de Información e Investigación; docente de postgrado en la Universidad Tecnológica Equinoccial. Ha publicado diferentes obras: Hacia una educación de calidad. El proyecto de investigación: Cómo elaborar. Análisis cualitativo de información apoyado con software ATLAS.ti. Entre sus campos de interés se encuentra: Investigación cualitativa, análisis de contenidos de la información, gerencia. 